\title{
Model Impulse Response High Frequency \\ Communication Channel with the Identification of Parameters of Multipath
}

Vadim S. Revin, Aleksander M. Mezhuev, Aleksander V. Korennoi* Military Training and Research Center of the Air Force «Air Force Academy ft. Professor N.E. Zhukovsky and Y.A. Gagarin» 54a Starykh Bol'shevikov Str., Voronezh, 394064, Russia

Received 05.08.2015, received in revised form 12.10.2015, accepted 06.01.2016

The improved Markov phenomenological model impulse response of the high frequency communication channel are presented in this work. The model allows for the identification of authorized multipath components by analyzing the combinations of possible values of the correlation parameters. Thus obtained impulse response with fixed parameters of the statistical model generates adequate communication channel during each period of quasi-stationary.

Keywords: model, multipath, identification, Markov process, the impulse response.

Citation: Revin V.S., Mezhuev A.M., Korennoi A.V. Model impulse response high frequency communication channel with the identification of parameters of multipath, J. Sib. Fed. Univ. Eng. technol., 2016, 9(3), $385-391$. DOI: 10.17516/1999-494X-2016-9-3-385-391.

\section{Модель импульсной характеристики}

декаметрового канала связи

с идентификацией параметров многолучевости

В.С. Ревин, А.М. Межуев, А.В. Коренной ВУНЦ ВВС «ВВА им. проф. Н.Е. Жуковского и Ю.А. Гагарина» Россия, 371600, Воронеж, ул. Старых Большевиков, 54 а

В статье рассмотрена усовершенствованная марковская феноменологическая модель импульсной характеристики декаметрового канала связи. Модель позволяет осушествлять идентификацию разрешенных компонентов многолучевости путем анализа комбинаций возможных значений параметров корреляции. Полученная таким образом импульсная характеристика с фиксированными статистическими параметрами формирует адекватную модель канала связи в течение каждого периода квазистационарности.

(C) Siberian Federal University. All rights reserved

* Corresponding author E-mail address: multitenzor@mail.ru 
Ключевые слова: модель, многолучевость, идентификаиия, марковский процесс, импульсная характеристика.

\section{Введение}

В радиосистемах между передатчиком и приемником часто возникают многолучевые непрямые трассы. Такое многолучевое распространение особенно значительно в декаметровом канале связи и обусловлено неоднородным отражением от слоев ионосферы. В результате принимаемый сигнал состоит из нескольких компонентов с различными временными задержками, амплитудами, фазами и направлениями прихода.

Следовательно, для улучшения рабочих показателей систем связи декаметрового диапазона необходимо обладать информацией об условиях распространения сигнала в канале, чтобы иметь возможность адаптировать к ним алгоритмы приема с целью обеспечения оптимальной обработки сигнала. Для этого необходимо проводить зондирование канала связи, т. е. осуществлять передачу по нему некоторого заранее известного сигнала, прием и обработка которого позволяют оценить параметры канала.

Анализ последних исследований и публикаций [1-3] показал, что поскольку набор случайных и детерминированных факторов, оказывающих существенное влияние на передачу сигналов в декаметровом канале связи, разнообразен, то целесообразно при его моделировании использовать феноменологический подход, так как соответствующие структурно-физические модели из-за своей сложности малопригодны для синтеза алгоритмов приема сигналов. При этом для феноменологических моделей канал рассматривается как «черный ящик», внутренняя структура которого при описании не учитывается, а основное внимание уделяется воспроизведению взаимозависимостей между входными и выходными воздействиями.

Цель работы заключается в построении модели канала связи, которая в отличие от наиболее часто применяемых (конкретизированных) моделей позволяет представить его на основе его импульсной характеристики с использованием теории марковских случайных процессов.

\section{Постановка задачи}

Так как свойства большинства реальных сред распространения радиоволн становятся нелинейными только при очень мощных сигналах, не характерных для радиосвязи, то декаметровый канал можно рассматривать как линейную и узкополосную систему.

Тогда представим в фиксированный момент времени реализацию его импульсной характеристики по модулю в виде [4, 5]

$$
\begin{aligned}
& \left|\begin{array}{ll}
H & \tau
\end{array}\right|=\left[\begin{array}{lllll}
h_{m} & \tau \cos \omega_{0} \tau-\varphi & \tau
\end{array}\right] \text { rect } \tau / \tau_{\max }= \\
& =\left[\begin{array}{lllll}
h_{c} & \tau & \cos \omega_{0} \tau+h_{s} & \tau & \sin \omega_{0} \tau
\end{array}\right] \text { rect } \tau / \tau_{\max },
\end{aligned}
$$

где $h_{m}(\tau), h_{c}(\tau)$ и $h_{s}(\tau)$ - огибающая и квадратурные составляющие импульсной характеристики; $\omega_{0}$ - несущая частота, соответствующая декаметровому диапазону; $\varphi(\tau)$ - фазовый сдвиг, отражающий характер фазовых искажений в канале, обусловленных расположением областей отражения в ионосферной линии связи и доплеровским смещением частоты; $\tau_{\max }-$ максимальное время задержки сигнала или «память» канала;

$$
-386-
$$




$$
\operatorname{rect} \frac{\tau}{\tau_{\max }}=\left\{\begin{array}{l}
1 \text { при } 0 \leq \tau \leq \tau_{\max } \\
0 \text { при } \tau<0 \text { и } \tau>\tau_{\max }
\end{array} .\right.
$$

Принимая во внимание результаты экспериментальных исследований прохождения радиоволн в ионосфере с учетом многолучевости и замираний [6], в [7] предложена следующая модель квадратурных составляющих $h_{c}(\tau)$ и $h_{s}(\tau)$ :

$$
\begin{aligned}
& h_{c} \tau=A_{c} c h \gamma_{c} \tau+m_{c}, \\
& h_{s} \tau=A_{s} \operatorname{sh} \gamma_{s} \tau+m_{s},
\end{aligned}
$$

которая вполне адекватно отражает свойства реальных декаметровых каналов связи, что подтверждается высокой степенью соответствия ее статистических характеристик экспериментальным данным.

Здесь $A_{c(s)}$ - постоянный коэффициент; $m_{c(s)}-$ величины, определяющие математическое ожидание $h_{c(s)}(\tau), \gamma_{c(s)}(\tau)$ - независимые (в общем случае векторные) гауссовские марковские случайные процессы, задаваемые стохастическим дифференциальным уравнением вида

$$
\frac{d \gamma_{c s} \tau}{d \tau}=-\beta_{c s} \gamma_{c s} \tau+\beta_{c s} n_{c s} \tau,
$$

где $\beta_{c(s)}$ - переменный параметр, обратный времени корреляции.

Для упрощения расчетов примем $A_{c(s)}=1 ; m_{c(s)}=0$.

При моделировании целесообразно в стохастическом дифференциальном линейном уравнении (4) перейти от непрерывного времени к дискретному. Для этого отрезок $\left[0, \tau_{\max }\right]$ разобьем $n$ эквидистантно расположенными точками $t_{v}=v \Delta$, где $\Delta=\tau_{\text {max }} / n-$ шаг дискретизации по времени, $v=0,1, \ldots, n$. Для достаточно точной оценки результатов зададим $n=1000 ; \tau_{\max }=1 \mathrm{мc}$ согласно [6].

Тогда для выражения (4) разностное уравнение принимает вид

$$
\gamma_{(v+1) c s}=\exp -\beta_{c s} \Delta \gamma_{v c s}+D_{c s}^{1 / 2} \Delta n_{v c s},
$$

где $n_{v c(s)}$ - белый гауссовский шум формирования, в качестве которого в модели взяты реализации датчика гауссовских случайных чисел.

Дисперсия белого гауссовского шума равна

$$
D_{c s} \Delta=\beta_{c s}{ }^{2} N_{0 c s} / 4 \beta_{c s} \quad 1-\exp -2 \beta_{c s} \Delta,
$$

где $N_{0 c(s)}$ - односторонняя спектральная плотность БГШ.

\section{Описание основных результатов исследования}

Вычислив дисперсию белого гауссовского шума согласно (6), задаваясь начальным значением $\gamma_{c(s)}(0)=0$, реккурентно вычисляем $\gamma_{(v+1) c(s)}$ по $\gamma_{v c(s)}$ в соответствии с выражением (5). Полученные таким образом значения $\gamma_{c(s)}$ представлены в виде графиков на рис. $1 a, \sigma$, при этом амплитуда $\gamma_{c(s)}$ нормировалась К $\sqrt{D_{c(s)}}$.

$$
-387-
$$




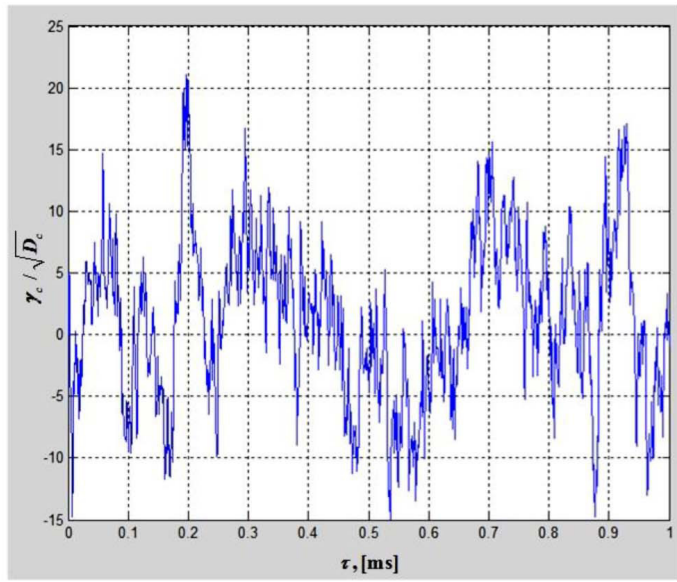

a)

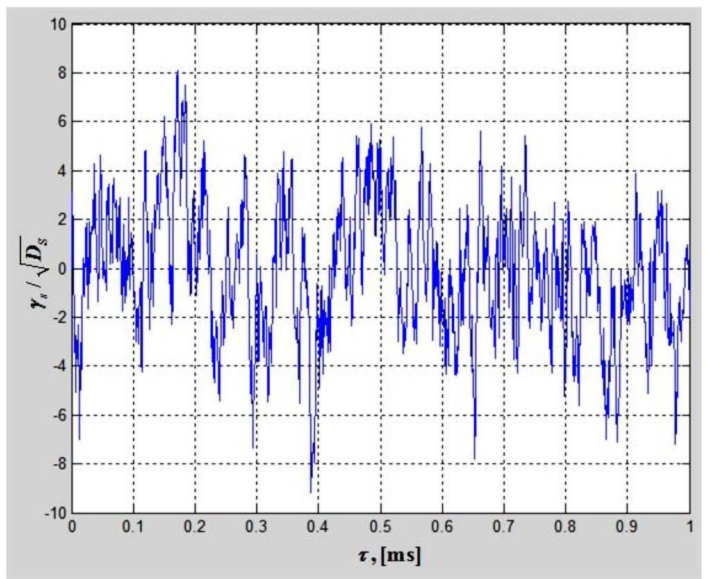

б)

Рис. 1. Графики независимых гауссовских марковских случайных процессов $\gamma_{c(s)}$

С учетом того, что преобразование (4) является невырожденным, нелинейным, $h_{c}(\tau)$ и $h_{s}(\tau)$ также будут марковскими процессами [8].

Затем, подставляя полученные результаты из (5) в (2) и (3), находим квадратурные составляющие импульсной характеристики $h_{c}(\tau)$ и $h_{s}(\tau)$. Результаты моделирования представлены в виде графиков на рис. $2 a, \sigma$.

Так как характер замираний в декаметровом канале постоянно меняется, процессы $h_{c}(\tau)$ и $h_{s}(\tau)$, очевидно, можно считать стационарными только на непродолжительных интервалах времени, соответствующих замираниям, описываемым определенным законом распределения с постоянными параметрами. Поэтому в общем случае параметры формирующего фильтра (4) будут зависеть от текущего значения времени. Согласно экспериментальным данным [9] период квазистационарности декаметрового канала связи составляет $\tau_{c}=5-15$ мин.

Затем, подставляя полученные результаты из (2), (3) в (1), вычисляем импульсную характеристику канала связи. Амплитуда выбросов импульсной характеристики и ее квадратурных составляющих нормировалась к среднеквадратическому отклонению $\sigma$.

Основное требование к выбору и обоснованию математической модели декаметрового канала связи определяется величиной априорной погрешности моделирования, возникающей вследствие неадекватности реализуемой модели статистическим характеристикам реального канала связи.

Поскольку типичный декаметровый канал связи в точке приема согласно экспериментальным данным [10] преимущественно имеет 2-4 луча распространения, то в рассматриваемой модели предлагается из всех возможных компонентов многолучевости учитывать только те, которые являются существенными, что определяется их пересечением выбранного соответствующим образом порогового уровня.

Количество разрешенных компонентов многолучевости $K_{p e r}$ может быть идентифицировано из функции модуля импульсной характеристики (1) с учетом установленного порогово- 


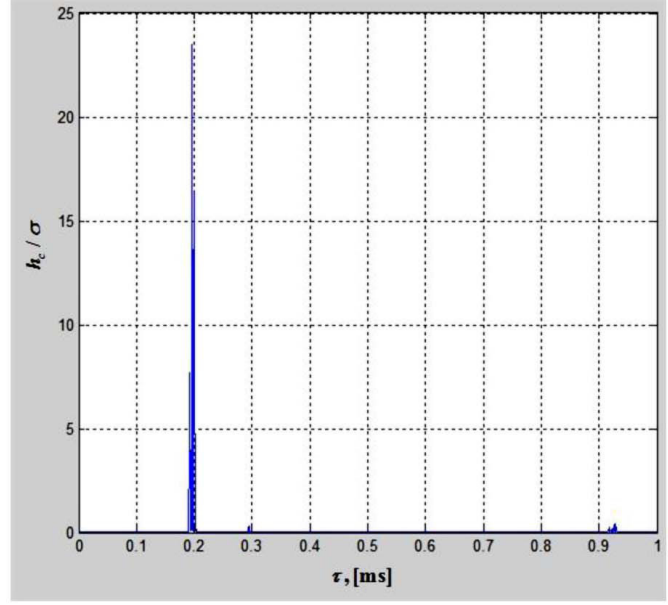

a)

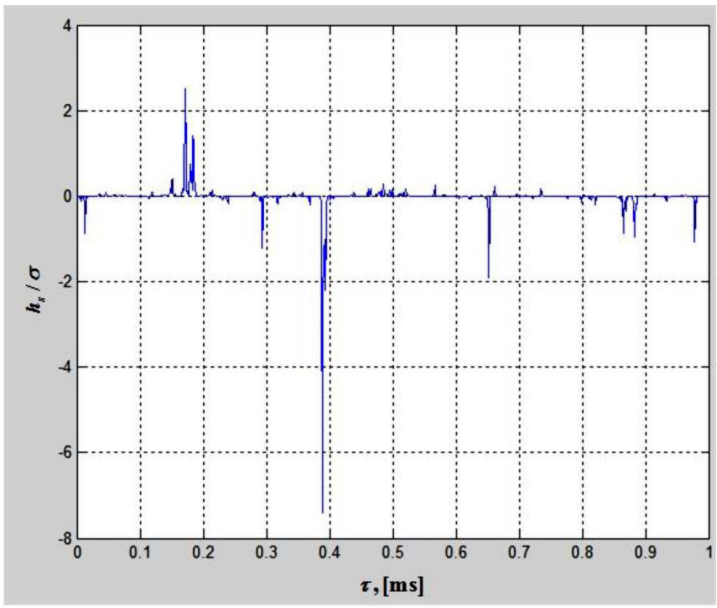

б)

Рис. 2. Графики квадратурных составляющих импульсной характеристики $h c(s)(\tau)$

го уровня. Рекомендованное значение порога, используемого для идентификации количества компонентов многолучевости, зависит от динамического диапазона измерительного оборудования и составляет уровень 15 дБ от максимального значения амплитуды импульсной характеристики [11].

На рис. $3 a$ и $б$ представлены графики импульсной характеристики для одной из реализаций без учета порога идентификации и с учетом порога идентификации компонентов многолучевости соответственно.

Для выбора адекватной модели импульсной характеристики производим анализ комбинаций возможных значений параметров корреляции $\beta_{c(s)}$. Для каждой такой комбинации вычисляем вероятность пересечения заданного порогового уровня 2-4 выбросами импульсной характеристики. Требуемое значение такой вероятности составляет $P_{p e r} \geq 0,9$.

Результаты моделирования, изображенные в виде объемной диаграммы на рис. 4 , позволили установить значения параметров корреляции, при которых обеспечивается выполнение определенных выше требований к адекватности модели $\beta_{c}=4$ и $\beta_{s}=25$ с $\mathrm{P}=0,91$.

\section{Заключение}

Таким образом, в статье рассмотрена усовершенствованная марковская феноменологическая модель импульсной характеристики декаметрового канала связи. Модель позволяет осуществлять идентификацию разрешенных компонентов многолучевости путем анализа комбинаций возможных значений параметров корреляции. Полученная таким образом импульсная характеристика с фиксированными статистическими параметрами формирует адекватную модель канала связи в течение каждого периода квазистационарности.

В дальнейших исследованиях предлагается полученную модель импульсной характеристики использовать для формирования на ее основе субоптимального фильтра, обеспечивающего обработку сигналов в декаметровом канале связи в условиях многолучевости.

$$
-389-
$$




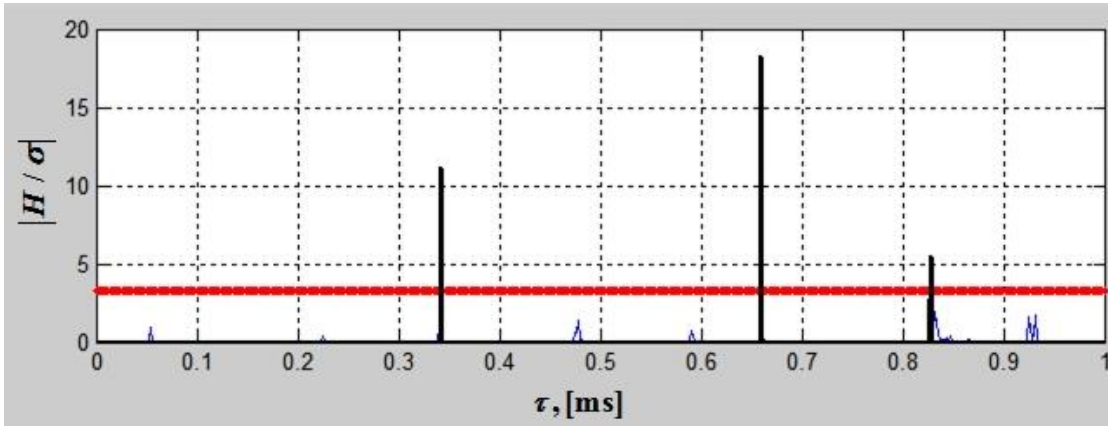

a

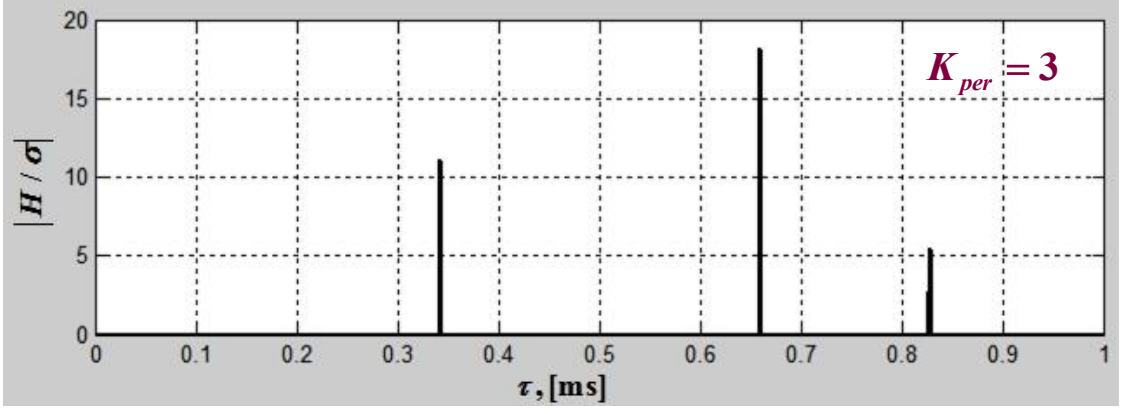

6

Рис. 3. График импульсной характеристики: а - без учета порога идентификации; б - с учетом порога идентификации

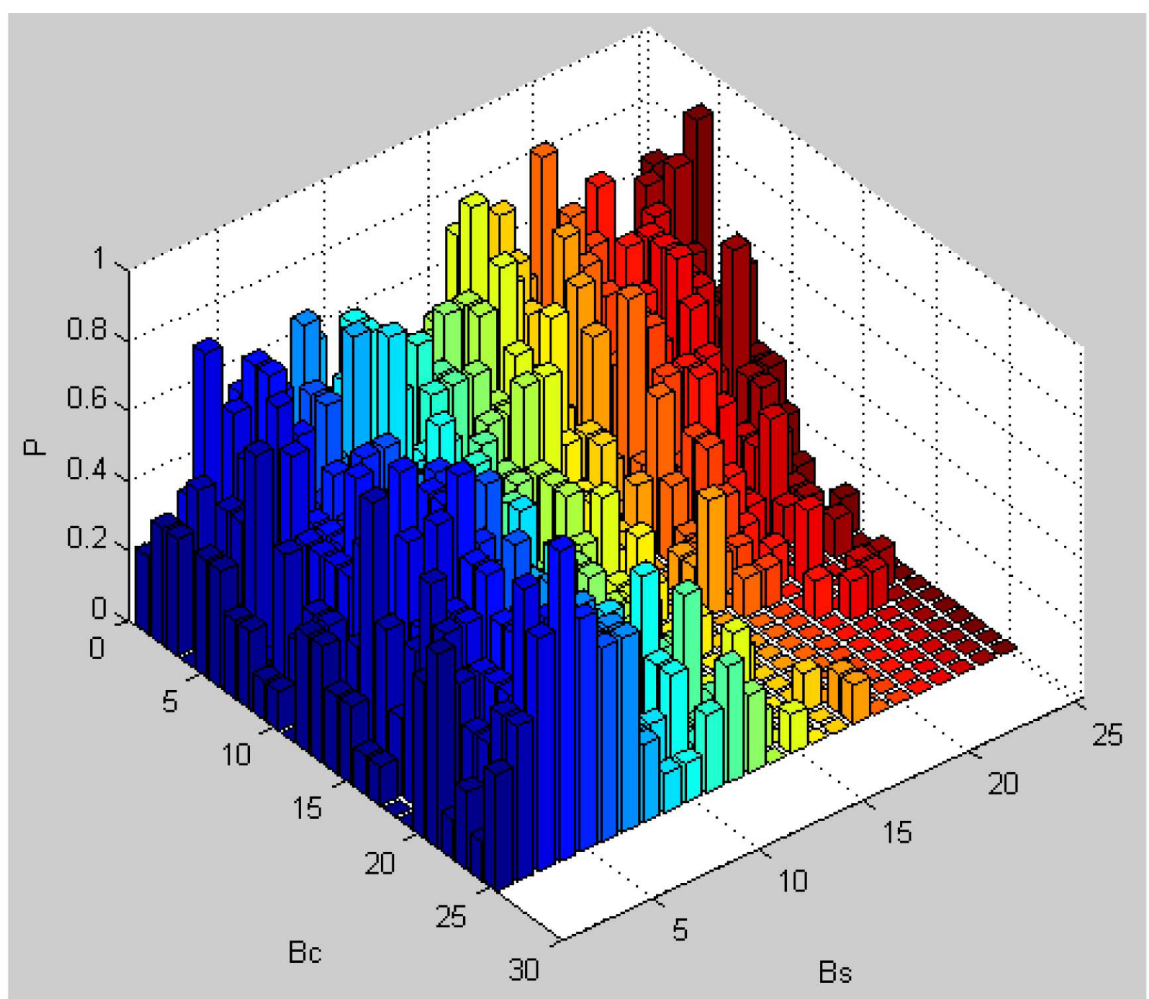

Рис. 4. Диаграмма проверки адекватности модели при различных комбинациях $\boldsymbol{\beta}_{c(s)}$ 


\section{Список литературы}

[1] Irshad Ya. On Some Continuous-time Modeling and Estimation Problems for Control and Communication. dissertation. Karlstad University Studies. 2013. 86 p.

[2] Kaiser T., Zheng F. Ultra wideband systems with MIMO. Chichester: John Wiley \& Sons Ltd, 2010. $254 \mathrm{p}$.

[3] Батенков К.А. Математическое моделирование непрерывных многопараметрических каналов связи в операторной форме. Телекоммуникации. 2013. № 10. C. 2-4. [Batenkov V.A. Mathematical modeling of continuous multivariable communication channels in the operator form. Telecommunications. 2013. № 10. 2-4. (in Russian)]

[4] Кловский Д.Д., Конторович В.Я., Широков С.М. Модели непрерывных каналов связи на основе стохастических дифференциальных уравнений. М.: Радио и связь, 1984. 247 с. [Klovsky D.D., Kontorovich V.J., Shirokov S.M. Models of continuous communication channels based on stochastic differential equations. M .: Radio and Communications, 1984. 247 (in Russian)]

[5] Тихонов В.И. Статистическая радиотехника. Изд. 2. М.: Радио и связь, 1982. 624 с. [Tikhonov VI Statistical radios. Ed. 2. M .: Radio and Communications, 1982. 624 (in Russian)]

[6] Долуханов М.П. Флуктуационные процессы при распространении радиоволн. М: Связь, 1971. С. 55-155. [Dolukhanov M.P. Fluctuation processes during propagation. M: Communications, 1971, 55-155. (in Russian)]

[7] Ершов Л.А., Коренной А.В., Шелковников М.А. Марковская модель декаметрового канала связи. Радиотехника. Обработка сигналов и полей. 1998. №1. C. 7-10. [Ershov L.A., Native A.V., Shelkovnikov M.A.,. Markov model decametre link. Radio engineering. Signal and fields Processing. 1998. №1. 7-10. (in Russian)]

[8] Тихонов В.И., Кульман Н.К. Нелинейная фильтрация и квазикогерентный прием сигналов. М:. Сов. радио, 1975. 704 с. [Tikhonov V.I., Kuhlman N.K. Nonlinear filtering and quasicoherent signal reception. M :. Soviet radio, 1975. 704 (in Russian)]

[9] Пат. 2388146 Российская Федерация. Брянцев В.Ф. Способ измерения амплитудно-частотных характеристик ионосферных каналов связи: 2010, Бюл. № 12. С. 17. [Bryantsev V.F. A method for measuring the amplitude-frequency characteristics of the ionospheric communication channels: Patent 2388146, Russian Federation. 2010, №12, 17. (in Russian)]

[10] Recommendation ITU-R F.1487 (05/2000). Testing of HF modems with bandwidths of up to about $12 \mathrm{kHz}$ using ionospheric channel simulators. ITU, 2000. P. 2-13.

[11] Recommendation ITU-R P.1407-5 (09/2013). Multipath propagation and parameterization of its characteristics. ITU, 2013. P. 6-23. 